\title{
Probing of exotics structure with hadron and heavy ion collisions
}

\section{Mikhail Barabanov}

JINR

Joliot-Curie 6, Dubna, Russia

E-mail: barabanovejinr.ru

\section{Alexander Vodopaynov}

JINR

Joliot-Curie 6, Dubna, Russia

E-mail: barabanovejinr.ru

The paper aims to selected studies of heavy exotic hadrons called X, Y, Z states. The topic includes detailed analysis of their strong, weak and electromagnetic decays containing $c \bar{c}$ pair, physics simulations and events reconstruction at NICA SPD/MPD.

The 39th International Conference on High Energy Physics (ICHEP2018)

4-11 July, 2018

Seoul, Korea 


\section{Introduction}

The analysis of X, Y, Z states is of great importance. Their predictions are closely linked to existing and forthcoming data of running and planned experiments like Belle, BaBar, BES, LHCb, NICA, PANDA, etc.. Given the existing experience of model calculation, physics simulation and event reconstruction the detailed analysis of structure of exotic states should be performed. This can be solved by using well known methods based on QCD principles as well as new proposed phenomenological approaches able to describe the structure of bound state of hadrons and exotics. The obtained results will shed light on the nature of X, Y, Z exotics, which are one of the most mysterious states in modern particle physics [1-4]. Chamonium-like exotics spectroscopy represents a good testing tool for the theories of strong interactions, including: QCD in both the perturbative and non-perturbative regimes, LQCD, potential models and phenomenological models. The experiments planned at NICA are well suited to testing these states. The facility will be able collide heavy ion beams with luminosity $10^{27} \mathrm{~cm}^{-2} \mathrm{c}^{-1}$ and $V_{\mathrm{s}}$ up to $11 \mathrm{GeV}$ and proton-proton beams with luminosity up $10^{32} \mathrm{~cm}^{-2} \mathrm{c}^{-1}$ and $\sqrt{\mathrm{s}}$ up to $26 \mathrm{GeV}$ [5].

\subsection{Heavy flavour study}

Good tracking and particle identification performance of NICA SPD over significant fraction of the final state phase-space can provide a good opportunity to extend its ambitious physics program to studies of heavy charmed objects via their decays to electrons, hadrons or photons. It can be illustrated by the PYTHIA8 [6] simulated data on heavy quarkonia production in p-p collisions at $\sqrt{s}_{\mathrm{s}} \sim 26 \mathrm{GeV}$. One can see from Fig. 1 that the detector acceptance for electron-positron pairs from $\mathrm{J} / \psi$ decays is quite high $(\sim 80 \%$ for $|\eta|<1.5)$. The detector acceptance for photons from electromagnetic decays of heavy quarkonia can be seen in Fig. 2. It exceeds $80 \%$ for $\chi_{\mathrm{c} 1}$ and $\chi_{\mathrm{c} 2}$ decays. The momentum resolution for electrons and photons is presented in Figs. 3, 4. One can see that it will be possible to combine momentum measurement of electrons in tracking detectors with energy measurement in the EMC to obtain better accuracy for fast particles. Signal reconstruction performance of the detector for $J / \psi \rightarrow \mathrm{e}^{-} \mathrm{e}^{+}$ decays was evaluated using full simulation/reconstruction chain. As can be seen in Fig. 5, J/ $\psi$ invariant mass peak is clearly visible.
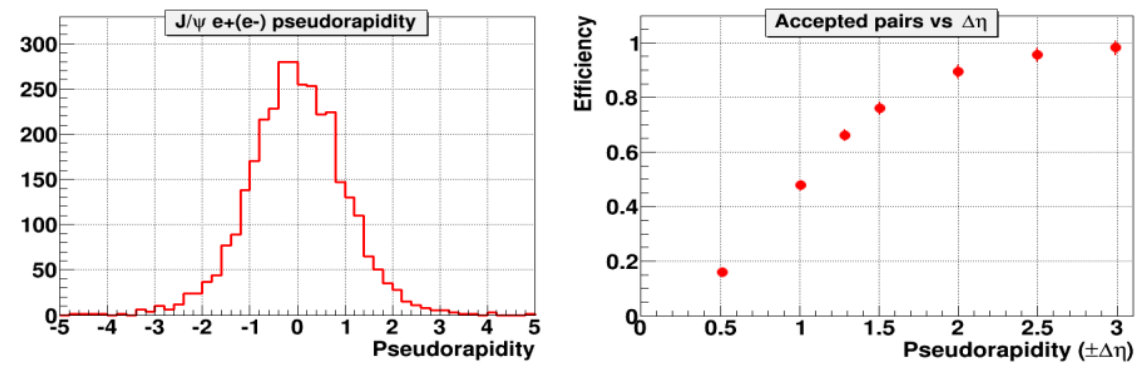

Fig. 1: Left) Pseudorapidity distribution of electrons and positrons from $\mathrm{J} / \psi$ decays; right) percentage of $\mathrm{J} / \psi \rightarrow \mathrm{e}^{-} \mathrm{e}^{+}$decays with both the electron and positron within the pseudorapidity window of $\pm \Delta \eta$. 

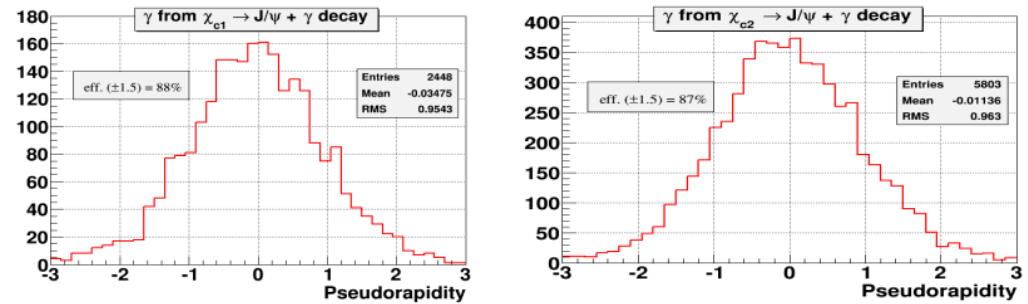

Fig.2. Pseudorapidity distributions of the photons from charmonia decays: left) $\chi_{\mathrm{c} 1}$, right) $\chi_{\mathrm{c} 2}$.
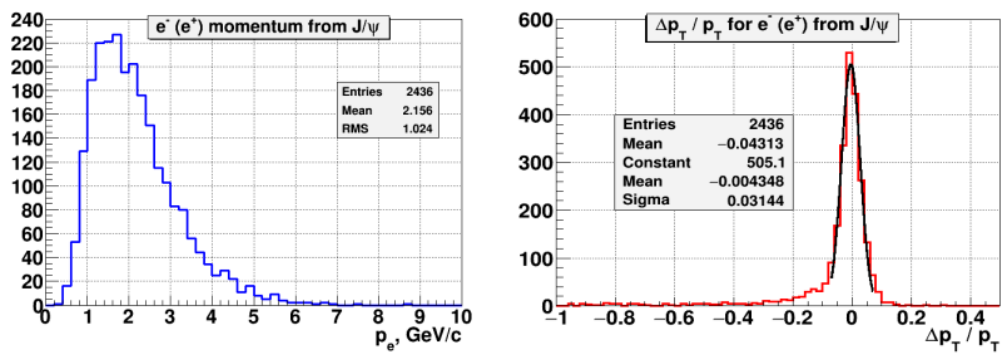

Fig. 3 Left) Momentum spectrum of electrons and positrons with $|\eta|<1.5$ from J/ $\psi$ decays; right) $\Delta \mathrm{p}_{\mathrm{T}} / \mathrm{p}_{\mathrm{T}}$ - distribution for decay products, where $\Delta \mathrm{p}_{\mathrm{T}}$ is the difference between reconstructed and true transverse momentum.
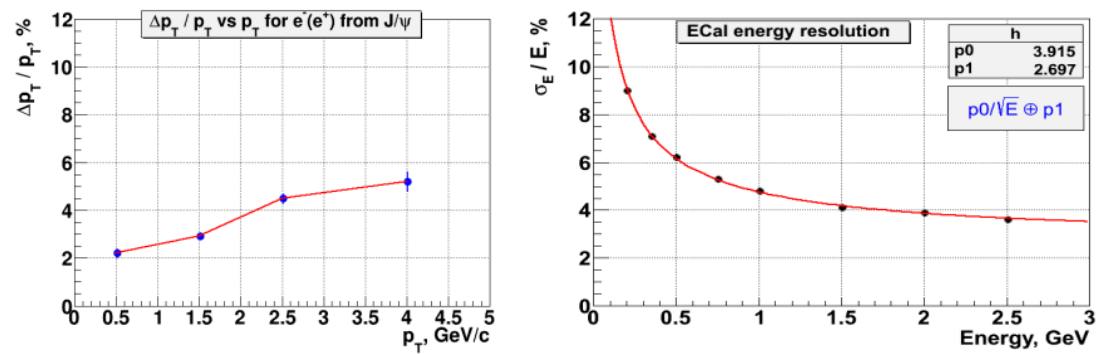

Fig. 4. Left) $\Delta \mathrm{p}_{\mathrm{T}} / \mathrm{p}_{\mathrm{T}}$ versus $\mathrm{p}_{\mathrm{T}}$ for electrons and positrons with $|\eta|<1.5$ from $\mathrm{J} / \psi$ decays; right) energy resolution $\left(\sigma_{\mathrm{E}} / \mathrm{E}\right)$ of the EMC for photons and electrons as a function of their energy.
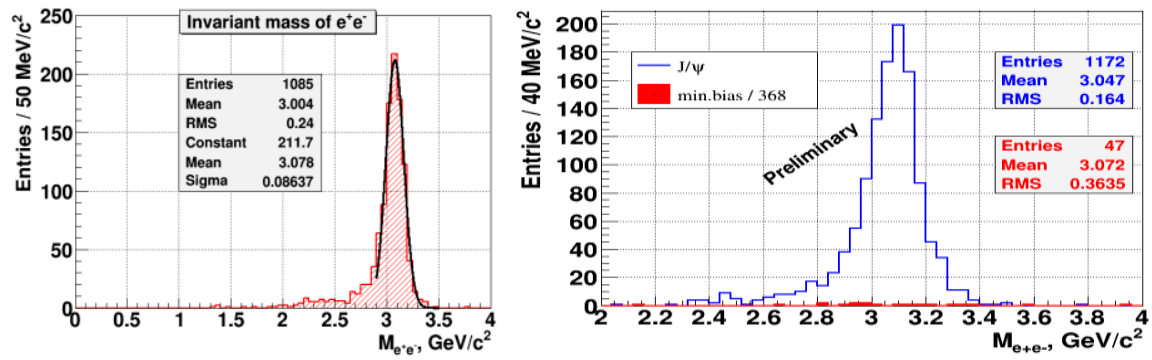

Fig. 5: Left - reconstructed invariant mass of electron-positron pairs from $\mathrm{J} / \psi$ decays (signal); right - the same and minimum bias events (background), remaining after applying selection cuts (red). Note that only $1 / 368$ part of all the minimum bias statistics was processed.

\subsection{Reconstruction of $\mathrm{X}(3872)$ exotics}

The exotic state $\mathrm{X}(3872)$ was simulated in PYTHIA8 under the assumption that it is a charmonium state and the branching ratio to $\mathrm{J} / \psi+\rho^{0}$ was taken to be $5 \%$ [7]. As a result, the 
$\mathrm{e}^{+} \mathrm{e}^{-} \pi^{+} \pi^{-}$final state branching ratio $\sim 3 \times 10^{-3}$ gives the cross section for this channel of $12.2 \mathrm{pb}$ or $\sim 10$ days of running time at the luminosity of $10^{32} \mathrm{~cm}^{-2} \mathrm{c}^{-1}$ to produce $\sim 1000$ events. To better distinguish the signal peak from the background it is better to use the invariant mass combination $\mathrm{Me}^{+} \mathrm{e}^{-} \pi^{+} \pi^{-}-\mathrm{Me}^{+} \mathrm{e}^{-}$due to its smaller width ( $10 \mathrm{MeV}$ in our case as can be seen in Fig. 6). Figure 6 also shows the background from events with charmonia production. The plots correspond to statistics collected during 10 months at luminosity of $10^{32} \mathrm{~cm}^{-2} \mathrm{c}^{-1}$. After fitting the background to the polynomial function using side bands of invariant mass distribution and subtracting it from the original distribution it is possible to observe a clear peak from the X(3872) decay (Fig. 7).

As an extension of this topic one can consider looking at the other decay modes $X(3872)$. Since the branching ratio of $\mathrm{X}(3872)$ to pairs of $\mathrm{D}$-mesons is much higher $\left(\mathrm{D}^{+} \mathrm{D}^{-}\right.$is $\sim 40 \%$ and $\mathrm{D}^{0} \mathrm{D}^{*}$ lbar is $\sim 55 \%$ ) one should try to evaluate the possibility to reconstruct this state from hadronic decays of D-meson pairs. For such a study, the ability to tag the D-meson decays using the silicon microvertex detector should be very important. Thus, this physics topic becomes synergetic to the heavy ion charm program of NICA.
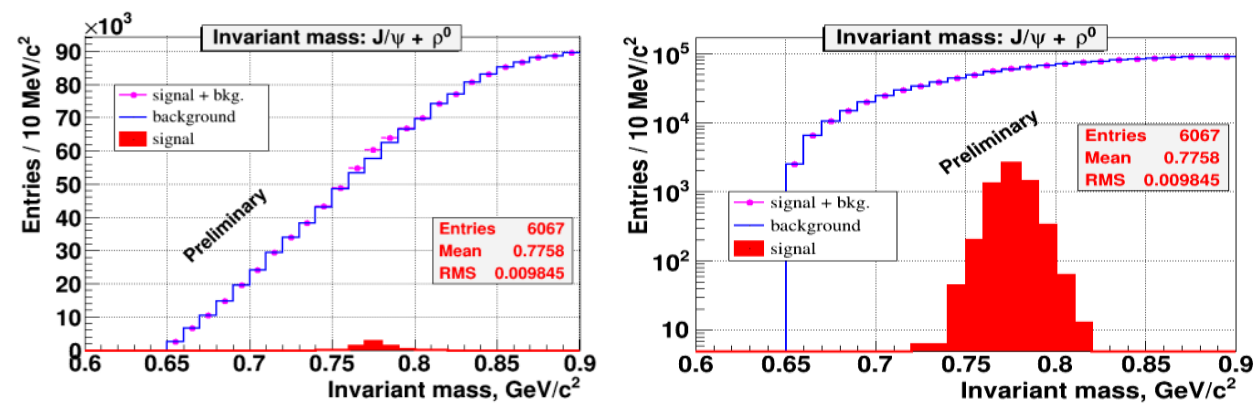

Fig. 6. Invariant mass combination $\mathrm{Me}^{+} \mathrm{e}^{-} \pi^{+} \pi^{--} \mathrm{Me}^{+} \mathrm{e}^{-}$.
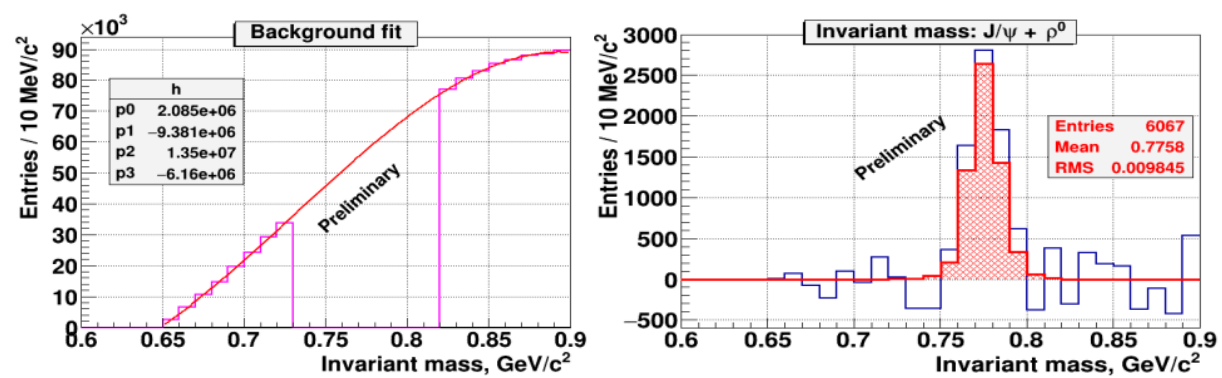

Fig. 7. Left - Background estimation using the polynomial fit of the side bands of Fig. 6 (left); right - background - subtracted invariant mass combination from Fig. 6 (left) (blue line) and true $\mathrm{X}(3872)$ histogram (red line).

\section{References}

[1] N. Brambilla et al., European Physical Journal C 71:1534, (2011) 1

[2] C. Patrignani et al. (Particle Data Group), Chin. Phys. C, 40, 100001 (2016) and 2017 update

[3] M.Yu. Barabanov, A.S. Vodopyanov, S.L. Olsen, Phys. At. Nuc., V.77, N.1, (2014) 126

[4] M.Yu. Barabanov, A.S. Vodopyanov, S.L. Olsen, Phys. At. Nuc., V.79, N 1 (2016) 126

[5] http://nica.jinr.ru/files/NICA CDR.pdf

[6] http://home.thep.lu.se/ torbjorn/Pythia.html/

[7] R. Aaij et al., LHCb Collaboration, Eur. Phys. J. C73, 2462 (2013) 